accepted it (74.6\% of those offered). 238 patients accepted a research pack, with 105 returning questionnaire 1 (44.1\% response rate), and 26 returning questionnaire 2 (24.8\% of initial respondents). Questionnaire 1 (patients) - 40\% found it difficult or burdensome repeating information to HCPs, and almost 70\% were interested in a PHR to reduce repeating information. Questionnaire 2 (patients)- 74\% found it the passport easy to use, but only a few actually used it regularly. Some felt the passport helped conversations with family and HCPs about day-to-day and future care. Over 60\% stated the PHR was 'very' or 'quite' useful', and would recommend it to others. Questionnaire and focus group with HCPs indicated it was mainly patients and relatives who used the passport, not non-palliative HCPs. Half felt the passport was initially well received, but only $4(22 \%)$ felt it was used regularly by patients or their families. More found it useful to talk about facilitation discussion about future care compared with day-today care.

Conclusions While initial uptake of the passport was high, use appeared low. This evaluation does not support widespread use of a PHR in palliative care, but it may be helpful to individuals, especially earlier in their disease trajectory.

\section{MEASURING THE IMPACT OF END OF LIFE CARE QUALITY IMPROVEMENT (QI) PROJECTS}

S Kite, A Cracknell, A Hurlow, C Iwaniszak, C Pattison, E Rees, A Winfield. The Leeds Teaching Hospitals NHS Trust

\subsection{6/bmjspcare-2018-ASPabstracts. 143}

Aim To demonstrate the application of QI methodology to improving end of life care (EOLC), using improvement data (run charts/statistical process control charts (SPC)) to measure the impact of interventions.

Background QI methods are applied widely across healthcare. SPC and run charts are employed to demonstrate if interventions can lead to sustained and significant improvements. Identifying variables that suit themselves to measurement by repeated data points is more of a challenge for interpersonal aspects of care (such as palliative and EOLC) than for technical interventions (Conry M, 2012).

Methods QI methodology and measures were employed in two QI EOLC initiatives in a large acute NHS trust. The first was a multidisciplinary collaborative to improve the care of patients at risk of clinical deterioration, to reduce avoidable deterioration and/or inappropriate cardiopulmonary resuscitation. The second was an intervention led by the Palliative Care Team to improve the assessment and management of terminal agitation on designated wards. SPC and run charts were created for these initiatives, with baseline data pre-intervention and on-going data collection during the testing, implementation and sustainability phases.

Both initiatives were developed following identification of local need; were led by frontline teams and empowered ward level innovation.

Results In the first initiative SPC charts demonstrated sustained, significant $25 \%$ reductions in cardiac arrest calls across the trust, and on 14 pilot wards a $125 \%$ increase in patients with a treatment escalation plan and $75 \%$ increase in documented CPR decisions.
In the second initiative, run charts demonstrated statistically significant improvements in the rate of assessment, reassessment and evaluation of terminal agitation $(p<0.05)$. Routine review and dissemination of data with the frontline teams in these initiatives enhanced collaborative engagement, motivation and success.

Conclusion SPC and run charts can be used to measure the impact of interventions, and contribute to improvements in EOLC.

\section{UNMET NEEDS FROM MENTAL AND PHYSICAL HEALTH CO-MORBIDITIES: A PROSPECTIVE AUDIT IN COMMUNITY PALLIATIVE CARE}

Sarah Yardley, Monika Gorny. Central and North West London NHS Foundation Trust

10.1136/bmjspcare-2018-ASPabstracts. 144

Background Increasingly patients referred to community specialist palliative care (SPC) have multiple co-morbidities including psychiatric and mental health needs in addition to physical disease. Access to psychiatric services is variable. There is no national standard for community-based shared care in this context and both palliative and psychiatric professionals report challenges.

Methods This prospective audit aimed to:

- establish local need for community psychiatric liaison/shared working;

- identify unmet needs for quality improvement. Patients discussed in a SPC community multidisciplinary team meeting were screened for three months.

Inclusion criteria:

SPC staff had concerns about managing mental health needs; contact with psychiatric services within last year; current medication prescription for psychiatric disorder. Exclusion criteria: not meeting inclusion criteria and dementia if SPC considered this appropriately managed without psychiatric input.

Results In 182 case discussions (May-July 2017), 35 patients (new $n=19 / 96$, complex $n=16 / 86$ ) with unmet mental health needs were identified. 26 had a formal psychiatric diagnosis; 12 had on-going psychiatric care. 20 had never had contact with a local community mental health team, 3 were previously discharged. SPC attempted shared care with mental health services in 10 cases ( 3 via GP, 1 patient refused, 1 via oncology psychology services, 4 received psychiatry services and 1 was accepted but died before being seen). Four patients' needs were first identified by psychiatric liaison teams during hospital admissions. SPC records demonstrate a further 17 patients whose current needs exceeded SPC expertise but no shared care or referrals were attempted. The remaining 4 patients needs were met directly by SPC. Exemplar vignettes with further learning will be shared.

Conclusions This demonstrates significant unmet mental health needs in our community SPC population. Addressing needs requires proactive liaison between SPC and mental health services with identification of best practice models that also integrate specialist services with general practice. 